\title{
Optimal Designs for Compartmental Models with Correlated Observations
}

\author{
J. LÓPEZ-FIDALGO, J. M. RODRÍGUEZ-DÍAZ, G. SÁNCHEZ \\ \& M. T. SANTOS-MARTÍN*
}

Facultad de Ciencias, Universidad de Salamanca, Spain

\begin{abstract}
The flow of internally deposited radioisotope particles inside the body of people exposed to inhalation, ingestion, injection or other ways is usually evaluated using compartmental models (see Sánchez \& López-Fidalgo, (2003, and López-Fidalgo \& Sánchez, 2005). The International Commission on Radiological Protection (ICRP, 1994) describes the model of the human respiratory tract, represented by two main regions. One of these, the thoracic region (lungs) is divided into different compartments. The retention in the lungs is given by a large combination of ratios of exponential sums depending on time. The aim of this work is to provide optimal times for making bioassays when there has been an accidental radioactivity intake and there is interest in estimating it. In this paper, a large two-parameter model is studied and a simplified model is proposed in order to obtain optimal designs in a more suitable way. Local c-optimal designs for the main parameters are obtained using the results of LópezFidalgo \& Rodríguez-Díaz, 2004). Efficiencies for all the computed designs are provided and compared.
\end{abstract}

Key Words: Bioassays, biokinetic models, design efficiencies, initial deposition factors, radioactivity retention

\section{Introduction}

Compartmental models are used to analyse a system by dividing it into a finite number of components, which are called compartments. The compartments interact with each other, exchanging different products, e.g. chemical substances, hormones, people from a population, etc. A compartmental model is a network where the nodes are compartments connected by arrows designing the flow of some substance from one to another. In particular, the flow and retention of some kind of "substance" will be considered in this paper. There are initial compartments where the intake (input) of the substance takes place and there are also final compartments from where the substance is eliminated (output). A general introduction to this theory can be found, for example, in Anderson (1983).

Let us consider a general compartmental model with compartments denoted by numbers, $i=1,2,3, \ldots, n+1$. This model will include the flow corresponding to the

*Correspondence Address: J. López-Fidalgo, Facultad de Ciencias, Universidad de Salamanca, Plaza de los Caídos s/n, 37002-Salamanca, Spain. Email: fidalgo@usal.es 
disintegrating rate of each compartment. Let $k_{i, j}$ be the rate of transfer from compartment $i$ to compartment $j$. For simplicity $k_{i}$ will be used instead of $k_{i, j}$ when there is not possible confusion. $q_{i}(t)$ be the retention in compartment $i$ at time $t$ and $b_{i}(t)$ the input coming from the environment to compartment $i$ at time $t$. The content at compartment $i$ can be represented by the equations:

$$
\begin{aligned}
\frac{\partial q_{i}(t)}{\partial t}= & \sum_{r} k_{r, i} q_{r}(t)-\sum_{j} k_{i, j} q_{i}(t)+b_{i}(t)=\sum_{r} k_{r, i} q_{r}(t)-k_{i} q_{i}(t) \\
& +b_{i}(t), \quad i=1, \ldots, n, \\
\frac{\partial q_{n+1}(t)}{\partial t}= & \sum_{r} k_{r, n+1} q_{r}(t) .
\end{aligned}
$$

If possible, a system will be decomposed in catenary unidirectional chains. A catenary unidirectional system is a sequence of compartments in such a way that each one receives flow from the previous one, with rate $k_{i-1}$, and gives flow to the next one, with rate $k_{i}$. The first one receives flow from the environment, $b_{1}(t)$, and the last one only gives flow to the environment with rate $k_{n}$. There can also be flow from each compartment $i$ to the environment given by rates $k_{i 0}, i=1,2, \ldots, n$. Figure 1 shows this situation. If the substance is a radioactive isotope the disintegrating rate will be taken into account given by a common rate, $\lambda_{R}$, for all compartments. The disintegration can be considered as a flow to the environment. Then, where it has been said flow to the environment it would be said flow to the environment or other compartments outside the catenary chain.

If the rates of transfer, $K_{i}=k_{i}+k_{i 0}+\lambda_{R}$, in a unidirectional catenary system are all different, then an explicit solution for the retention is derived straightforwardly for the case of an impulse (acute) input $b_{1}$ at time $t=0$ (Skrable, 1974),

$$
q_{i}(t)=b_{1}\left(\prod_{p=1}^{i-1} k_{p}\right) \sum_{j=0}^{i}\left(\frac{e^{-K_{j} t}}{\prod_{p=0, p \neq j}^{i}\left(K_{p}-K_{j}\right)}\right) e^{-\lambda_{R} t}, \quad i=1,2, \ldots, n
$$

where $\lambda_{R}$ is the radioactive decay constant of the isotope. If there is no radioactive isotope then $\lambda_{R}=0$.

Thus, it would be very convenient to decompose a system in catenary unidirectional chains, if possible.

Compartmental systems are usually described by using flow diagrams, and are widely used in medicine, chemistry pharmacokinetics, etc. In fact, the human body is usually seen and studied as an example of a compartmental model, divided into several regions. We are interested in one of them, the respiratory tract.

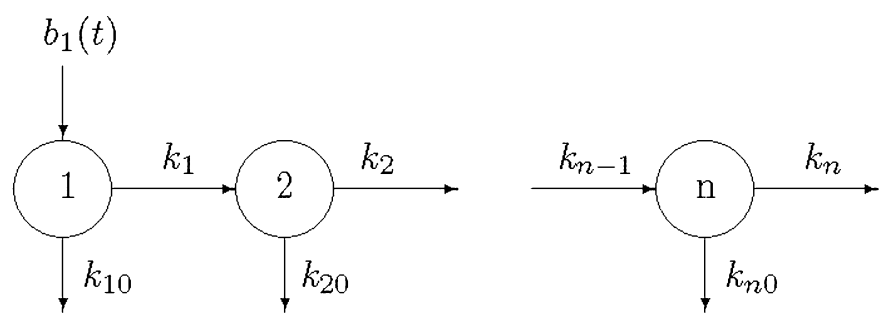

Figure 1. Catenary unidirectional system with n compartments 
ICRP 66 (1994) describes the compartmental model of the human respiratory tract applied to the intake of radioactive aerosols by inhalation. In this paper, the case of an intake of a radioactive substance in the human body will be considered. In particular, we will refer to the retention in the thoracic region (lungs) as a function of the time after an acute intake at $t=0$. The lung retention can be mathematically represented by a function $\eta(t, I, p),\left[\tau_{1}, \tau_{2}\right]$, where $I$ is the input to the system at $t=0$ and $p$ an index of the particle's size.

\section{Solving the Respiratory Tract Model}

The previous ideas have been applied to solve the respiratory tract model (ICRP 66, 1994) for the intake of radioactive aerosols by inhalation. For modelling purposes, the respiratory tract is represented by different regions (Figure 2): (i) The extrathoracic comprising the anterior nose $\left(E T_{1}\right)$ and the posterior nasal passage, larynx, pharynx and mouth $\left(E T_{2}\right)$, and (ii) the thoracic region that represents the lungs and it is divided into bronchial $(B B)$, bronchiolar $(b b)$ and alveolar-interstitial $(A I)$.

In the ICRP 66 (1994) model the material from environment is deposited in the respiratory tract in compartments labelled as Particles in Initial State (PIS), except in compartment $E T_{1}$ and the lymphatic nodes, represented by compartment 10 and 13 in Figure 3. From each PIS compartment the material is transferred into the body fluids, at an

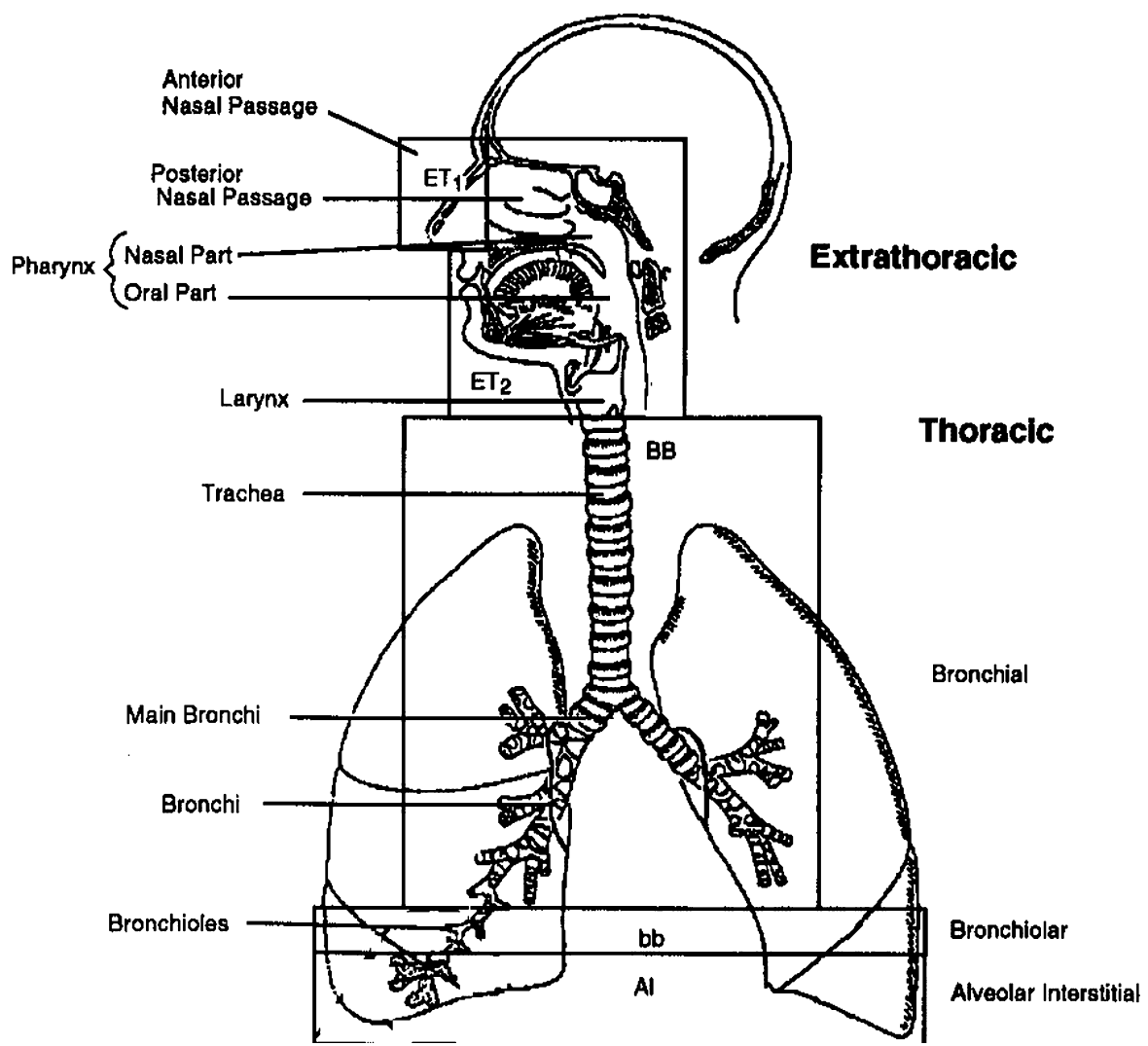

Figure 2. Anatomical regions of the respiratory tract 


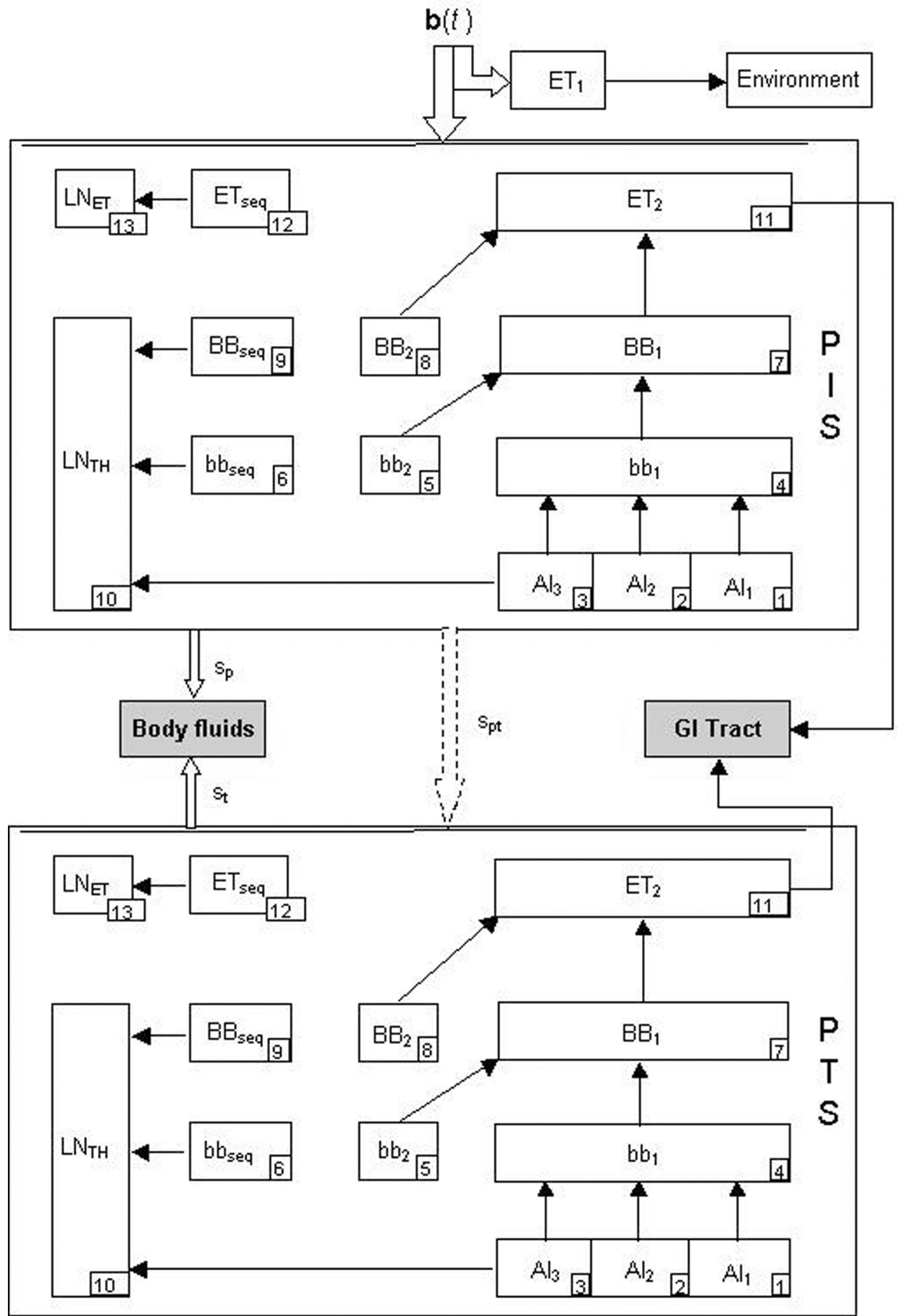

Figure 3. ICRP 66 (1994) Respiratory Tract Model. The dashed arrow from subsystem PIS to subsystem PTS means that the flow goes from each compartment in PIS to the compartment with the same number in PTS. The hollow arrow $(\Rightarrow)$ means a flow from each compartment in subsystem PIS or PTS to the "Body fluid". A simple arrow means flow from a single compartment to another 
absorption rate $s_{p}$. It is also simultaneously transferred from PIS (at a rate $s_{p t}$ ) to a corresponding compartment labelled as Particles in Transformed State (PTS). The flow goes from 1 in PIS to 1 in PTS, from 2 in PIS to 2 in PTS, and so on. We can consider that each compartment in PIS has a "mirror" compartment in PTS. In each compartment in PTS the isotope is dissolved at a constant rate $s_{p}$ into the body fluids (usually the blood). For instance, the total transfer rate for $A I_{2}$ in PIS will be $K_{A I_{2}}=k_{2,4}+s_{p t}+s_{p}$, and for $A I_{2}$ in PTS will be $K_{A I_{2}}^{\prime}=k_{2,4}+s_{t}$. This general model for the respiratory tract is common to any element. Standard clearance rates $k_{i j}$ are shown in Table 1 . The absorption rates $s_{p t}, s_{p}, s_{t}$ are related with the chemical form of the element. ICRP 66 (1994) establishes three types of materials according to its absorption behaviour: Fast (F), Moderate (M), and Slow (S). Default absorption rates are shown in Table 2.

If a person breaths a quantity $I$ at time $t=0$ the fraction of the airborne material that is deposited in each region is determined by the Activity Median Aerodynamic Diameter (AMAD), some anatomical and physiological parameters as well as various conditions of exposure. The AMAD is the median of the distribution of radio-labelled particles with varying activities and aerodynamic diameters and includes in its definition the size, shape and density of the particles. This is the parameter $p$ given in the introduction. We will assume standard anatomical and physiological values for a reference worker given in ICRP 66 (1994).

The fraction of $I$ from an environment deposited in PIS compartments $\left\{E T_{1}, 1, \ldots, 9,11,12\right\}$ - notice that no particles from environment were deposited directly either in compartments 10 and 13 or in PTS - is a function of $p$. They are the so-called Initial Deposition Factors, $I D F_{i}(p)$. The quantity $I D F_{i}(p)$ may be either calculated following the procedure described in ICRP 66 (1994) or obtained from Annex F of ICRP 66 (1994). Document ICRP 66 (1994) already gives the procedure to compute

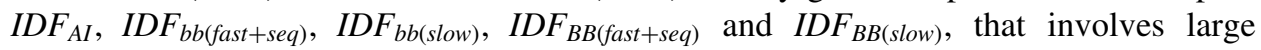
algebraic expressions. However we have found that the $I D F$ parameters, in the range of interest of AMAD, [0.5 $\mu \mathrm{m}, 20 \mu \mathrm{m}]$, for ordinary workers may be fitted using least squared estimators as follows:

$$
\begin{aligned}
I D F_{A I}(p) & =0.128187 e^{-0.170111 p} \\
I D F_{b b(\text { fast }+ \text { seq })}(p) & =0.0100737 e^{-0.0878945 p} \\
I D F_{b b(\text { slow })}(p) & =0.0212844 e^{-4.35327 p}+0.00920991 e^{-0.147244 p} \\
I D F_{B B(\text { fast }+ \text { seq })}(p) & =-0.0171738 e^{-0.566783 p}+0.0171738 e^{-0.0577835 p} \\
I D F_{B B(\text { slow })}(p) & =-0.0110839 e^{-1.11147 p}+0.0110839 e^{-123578 p}
\end{aligned}
$$

Using the expressions given in ICRP 66 (1994) the following functions $I D F_{i}(p)$ should

Table 1. Reference clearance rates for human respiratory tract

\begin{tabular}{lccccccc}
\hline Pathway & $k_{1,4}$ & $k_{2,4}$ & $k_{3,4}$ & $k_{3,10}$ & $k_{4,7}$ & $k_{5,7}$ & $k_{6,10}$ \\
Rate $\left(d^{-1}\right)$ & 0.02 & 0.001 & 0.0001 & 0.00002 & 2 & 0.03 & 0.01 \\
\hline & $k_{7,11}$ & $k_{8,11}$ & $k_{9,10}$ & $k_{11,15}$ & $k_{12,13}$ & $k_{E T 1}$, Enviroment & \\
& 10 & 0.03 & 0.01 & 100 & 0.001 & 1 & \\
\hline
\end{tabular}


1080 J. López-fidalgo et al.

Table 2. Default absorption rates for type F, M and S materials

\begin{tabular}{lccc}
\hline Absorption rate & $\mathrm{F}$ (Fast) & $\mathrm{M}$ (moderate) & $\mathrm{S}$ (Slow) \\
\hline$s_{p}\left(d^{-1}\right)$ & 100 & 10 & 0.01 \\
$s_{p t}\left(d^{-1}\right)$ & 0 & 90 & 100 \\
$s_{t}\left(d^{-1}\right)$ & - & 0.005 & 0.0001 \\
\hline
\end{tabular}

be used instead of $I D F_{A I}, I D F_{b b(f a s t+s e q)}, I D F_{b b(s l o w)}, I D F_{B B(f a s t+s e q)}$ and $I D F_{B B(s l o w)}$ :

$$
\begin{aligned}
& I D F_{1}(p)=0.3 I D F_{A I}(p) \\
& I D F_{2}(p)=0.6 I D F_{A I}(p) \\
& I D F_{3}(p)=0.1 I D F_{A I}(p) \\
& I D F_{4}(p)=0.993 I D F_{b b(\text { fast }+ \text { sec })}(p)-0.007 I D F_{b b(\text { slow })}(p) \\
& I D F_{5}(p)=I D F_{b b(\text { show })}(p) \\
& I D F_{6}(p)=0.007 I D F_{b b(\text { fast }+ \text { seq })}(p)+I D F_{b b(\text { show })}(p) \\
& I D F_{7}(p)=0.993 I D F_{B B(\text { fast }+ \text { sec })}(p)-0.007 I D F_{B B(\text { slow })}(p) \\
& I D F_{8}(p)=I D F_{B B(\text { slow })}(p) \\
& I D F_{9}(p)=0.007 I D F_{B B(\text { fast }+ \text { seq })}(p)+I D F_{B B(\text { slow })}(p)
\end{aligned}
$$

We are interested in evaluating the retention in the lungs for an acute intake $I$ at time $t=0$ for a worker exposed to aerosol particles of type $\mathrm{S}$. The lungs are represented by compartments 1 to 10 in PIS jointly with their "mirror" compartments in PTS. For evaluating the retention in each compartment this region can be divided in catenary branches (about 100 of them). Each catenary branch starts in some deposition compartment, (1 to 9) in PIS, and finishes in "Body fluids" or ET2. Notice that neither "Body fluids" nor ET2 are part of the lungs. For example, starting at compartment $5\left(b b_{2}\right)$ the following catenary branches may be built, where the prime means the corresponding "mirror" compartment:

$$
\begin{aligned}
b b_{2} & \rightarrow B \\
b b_{2} & \rightarrow B B_{1} \rightarrow B \\
b b_{2} & \rightarrow B B_{1} \rightarrow E T_{2} \\
b b_{2} & \rightarrow b b_{2}^{\prime} \rightarrow B \\
b b_{2} & \rightarrow b b_{2}^{\prime} \rightarrow B B_{1}^{\prime} \rightarrow B \\
b b_{2} & \rightarrow b b_{2}^{\prime} \rightarrow B B_{1}^{\prime} \rightarrow E T_{2}^{\prime} \\
b b_{2} & \rightarrow B B_{1} \rightarrow B B_{1}^{\prime} \rightarrow B \\
b b_{2} & \rightarrow B B_{1} \rightarrow B B_{1}^{\prime} \rightarrow E T_{2}^{\prime}
\end{aligned}
$$

Now equation (1) may be used to compute the content in all compartments of the thoracic region (lungs). The $k_{i j}$ values can be obtained from Table 1, and the absorption rate for type $\mathrm{S}$ from Table $2\left(s_{p t}=0.1, s_{p}=100, s_{t}=0.0001\right)$. Taking into account that $b_{1}=I \times I D F_{i}(p)$, where $I D F_{i}(p)$ is given by equation (2), these parameters can be replaced in the main equation obtaining the retention as a rational function of large 
sums of exponentials depending on $p$ and $t$, as follows:

$$
\eta(I, t, p)=I \frac{\sum_{i} \gamma_{i} e^{\alpha_{i} p+\beta_{i} t}}{\sum_{i} \gamma_{i}^{\prime} e^{\alpha_{i}^{\prime} p}}
$$

The full equation for the specified values of the parameters is shown in the Appendix. This equation may be obtained using the software BIOKMOD, provided by the third author (http://web.usal.es/ guillerm or the online version http://www3.enusa.es/ webMathematica/Public/biokmod.html).

The theoretical lung retention can be compared with experimental data using a lung counter, $y(t)$ measured along the time. The problem consists on estimating $I$ and $p$ efficiently taking the minimum number of measures $y(t)$. The model is linear in $I$ but not in $p$. The bigger the particles are, the more easily they are eliminated. The aim of this paper is to provide optimal times for making bioassays when there has been an accidental radioactivity intake and there is interest in estimating this. Usually the values of $p$ vary between 1 and 10 units. Figure 4 represents the initial model for $I=1000$ and $p=5$.

\section{Optimal Designs for the Original Model}

Let us assume there is only one worker. First, let us consider the case when two observations $y_{t_{1}}, y_{t_{2}}$ are taken from a worker at times $t_{1}$ and $t_{2}$ respectively. These are repeated measurements and need to be considered in the optimal design theory in a particular way taking into account the possible correlation between the observations. For a recent reference on these kind of problem see Müller \& Stehlík (2004). For the case considered here the following result comes easily.

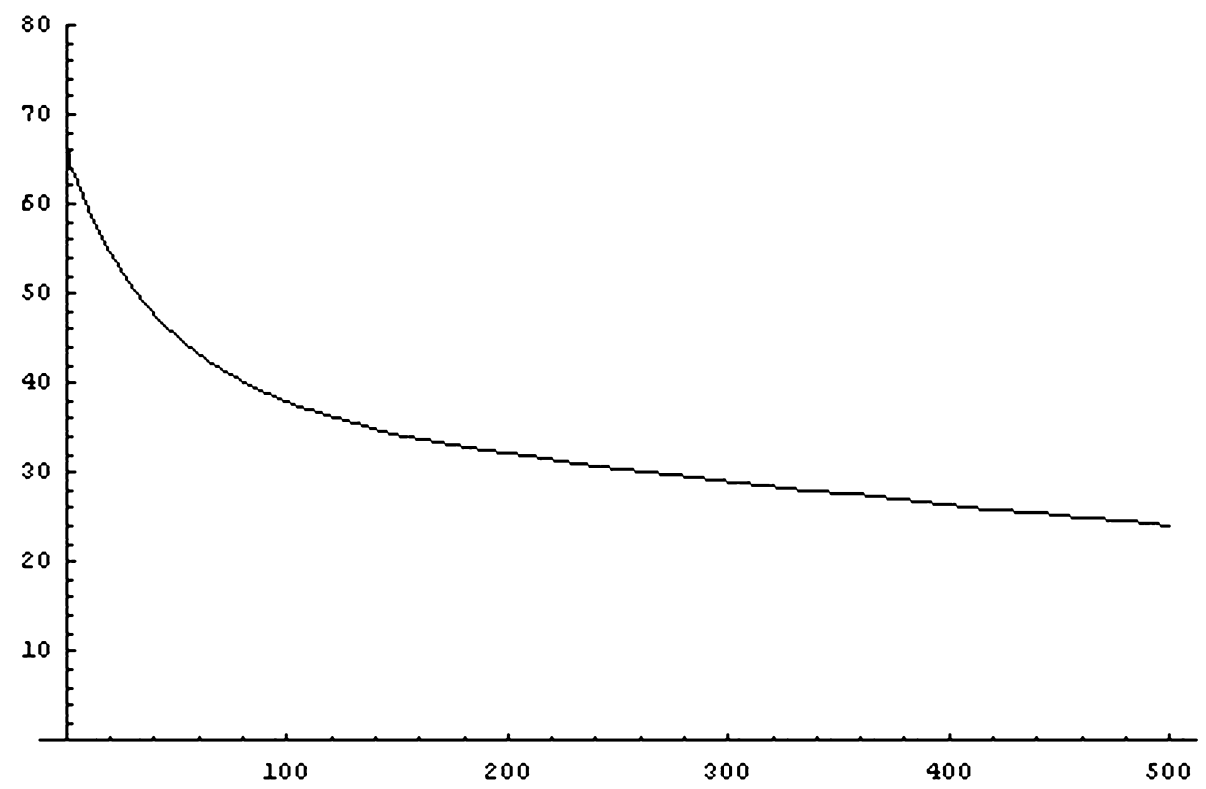

Figure 4. Lung's retention for $I=1000, p=5$ 


\section{Proposition}

Neither the $D$-optimal design depends on the linear parameter $I$ nor the $c$-optimal designs do for $c=c_{1}=(1,0)^{T}$ or $c=c_{2}=(0,1)^{T}$.

\section{Proof}

The model can be written as

$$
\eta(t, I, p)=I f_{1}(t, p)
$$

Denoting $f_{2}(t, p)=\partial f_{1}(t, p) / \partial p$, then

$$
\begin{aligned}
\nabla \eta(t, I, p) & =\left(\frac{\partial \eta(t, I, p)}{\partial I}, \frac{\partial \eta(t, I, p)}{\partial p}\right)^{T} \\
& =\left(f_{1}(t, p), I f_{2}(t, p)\right)^{T}=B(I)\left(f_{1}(t, p), f_{2}(t, p)\right)^{T}
\end{aligned}
$$

with

$$
B=B(I)=\left(\begin{array}{ll}
1 & 0 \\
0 & I
\end{array}\right)
$$

The information matrix is

$$
M=X^{T} \Sigma^{-1} X=B \tilde{X}^{T} \Sigma^{-1} \tilde{X} B=B \tilde{M} B
$$

where $\Sigma$ is the covariance matrix and $\tilde{M}=\tilde{X}^{T} \Sigma^{-1} \tilde{X}$ does not depend on $I$, where

$$
\tilde{X}=\left(\begin{array}{cc}
f_{1}\left(t_{1}, p\right) & f_{2}\left(t_{1}, p\right) \\
f_{1}\left(t_{2}, p\right) & f_{2}\left(t_{2}, p\right)
\end{array}\right)
$$

The proof finishes taking into account that

$$
\operatorname{det}(M)=\operatorname{det}(B)^{2} \operatorname{det}(\tilde{M}) \quad \text { and } \quad \Phi_{c}(M)=c^{T} M^{-1} c=\Phi_{B^{-1} c}(\tilde{M})
$$

This completes the proof.

Let us consider

$$
\Sigma=\sigma^{2}\left(\begin{array}{cc}
1 & e^{-\rho d} \\
e^{-\rho d} & 1
\end{array}\right)
$$

where the covariance between the two samples at times $t_{1}$ and $t$ depends on the distance between them, $d=t_{2}-t_{1}$. For D-optimality we look for two points $t_{1}=0$ and $t_{2}=d$ in the design interval maximizing $\operatorname{det} \tilde{M}$ for different values of $\rho$. For $c_{i}$-optimality similar designs are found minimizing the variance $c_{i}^{T} M^{-1} c_{i}$ for $c_{1}=(1,0)^{T}$ and $c_{2}=(0,1)^{T}$.

We will show the optimal designs for different criterion functions and several values for $\rho$. We consider also two different design intervals. The linearized model will be used, so that taking into account the previous result we can restrict the model to $\left(f_{1}(t, p), f_{2}(t, p)\right)^{T}$, that does not depend on $I$. The initial value for $p$ will be set to $p=5$. 


\section{First Observation at Zero}

Previous and posterior results lead us to fix the first observation at the very first possible moment in the interval $[0, \tau]$. For this interval the first time point is assumed $t=0$. The upper limit of the interval, $\tau$, will be considered as large as necessary in the computations. Table 3 shows the optimal times to take the second sample depending on the value of $\rho$ and the different optimization criteria.

\section{Delayed First Observation}

Actually the practitioner needs some time to start taking observations. This period must be at least 12 hours in our case, which moves the design interval to $[0.5, \tau]$. Again, when fixing the first observation at the beginning of the interval the second is shown in Table 4 for different values of $\rho$ and for several optimality criteria.

Tables 3 and 4 show that the smaller is $\rho$, the closer must be the two samples. In other words, when there is a strong relationship between observations the best design chooses them very close to each other.

Furthermore, there are big differences in choosing the best day to take the second observation for the two starting points $t=0$ and $t=0.5$. The explanation for the different results is that the model's curvature is specially concentrated at the very beginning, particularly in the interval $[0,1]$. This fact is especially clear in the geometric computation of the $c$-optimal designs using Elfving's (1952) method (see Figures 5 and 6). In particular Figure 6 shows one of the Elfving's set branches, increasing $t$ in 0.5 units each time. It can be seen that for $t>1$ the plot is almost a straight line.

\section{A Simplified Class of Model}

If we pay attention to the original model we can see that all the denominators of the fractions do not depend on $t$. This leads us to the model:

$$
\tilde{\eta}(I, t, p)=I \frac{\gamma_{1} e^{\alpha_{1} p+\beta_{1} t}+\gamma_{2} e^{\alpha_{2} p+\beta_{2} t}}{1+\gamma_{3} e^{\alpha_{3} p}}
$$

for specific values of $\alpha=\left(\alpha_{1}, \alpha_{2}, \alpha_{2}\right)^{T}$, and $\gamma=\left(\gamma_{1}, \gamma_{2}, \gamma_{3}\right)^{T}$.

We will focus on $D$-optimization. A general two-point design in $\left[\tau_{1}, \tau_{2}\right]$ will be $\xi=\{t, t+d\}$. Let us take $x=e^{t}, \theta=e^{p}$, thus $\xi$ takes the form $\{x, x \delta\}$, with $\delta=e^{d}$,

Table 3. Second observation's time for different values of $\rho$ when the first observation is fixed at $t=0$. The criteria used are D-optimality, $c_{1}$-optimality $\left(\phi_{c_{1}}\right)$ and $c_{2}$-optimality $\left(\phi_{c_{2}}\right)$

\begin{tabular}{lccc}
\hline$\rho$ & $D$ & $\phi_{c_{1}}$ & $\phi_{c_{2}}$ \\
\hline 0.001 & 0.14741 & 0.08762 & 0.05502 \\
0.01 & 0.20447 & 0.12837 & 0.09834 \\
0.1 & 0.20695 & 0.14016 & 0.14242 \\
1 & 0.23138 & 0.15572 & 0.16778 \\
10 & 2.19645 & 2.94087 & 2.81104 \\
100 & 2.19645 & 2.94087 & 2.81103 \\
1000 & 2.19645 & 2.94087 & 2.81103 \\
\hline
\end{tabular}


1084 J. López-fidalgo et al.

Table 4. Second observation's time for different $\rho$, first observation fixed at time $t=0.5$. The criteria used are D-optimality, $c_{1}$-optimality $\left(\phi_{c_{1}}\right)$ and $c_{2}$-optimality $\left(\phi_{c_{2}}\right)$

\begin{tabular}{lccc}
\hline$\rho$ & $D$ & $\phi_{c_{1}}$ & $\phi_{c_{2}}$ \\
\hline 0.001 & 1.15473 & 1.01307 & 0.96813 \\
0.01 & 1.15284 & 1.09390 & 1.09354 \\
0.1 & 1.16246 & 1.13000 & 1.13699 \\
1 & 69.0073 & 88.8826 & 88.0903 \\
10 & 69.0198 & 88.8826 & 88.0903 \\
100 & 69.0198 & 88.8826 & 88.0903 \\
1000 & 69.0198 & 88.8826 & 88.0903 \\
\hline
\end{tabular}

and the design interval comes to be $\left[e^{\tau_{1}}, e^{\tau_{2}}\right]$.The linearized model can be reduced to $\left(f_{1}(x, \theta), f_{2}(x, \theta)\right)^{T}$, with

$$
f_{1}(x, \theta)=\frac{\gamma_{1} \theta^{\alpha_{1}} x^{\beta_{1}}+\gamma_{2} \theta^{\alpha_{2}} x^{\beta_{2}}}{1+\gamma_{3} \theta^{\alpha_{3}}}, \quad f_{2}(x, \theta)=\frac{\partial f_{1}(x, \theta)}{\partial \theta}
$$

and for a general covariance matrix

$$
\Sigma=w\left(\begin{array}{ll}
1 & v \\
v & 1
\end{array}\right)
$$

where $v=\operatorname{cov}(t, t+d)$, the determinant can be expressed by

$$
\operatorname{det}[M(\xi)]=\frac{\left[f_{1}(x \delta, \theta) f_{2}(x, \theta)-f_{1}(x, \theta) f_{2}(x \delta, \theta)\right]^{2}}{w^{2}\left(1-v^{2}\right)}
$$

In the homoscedastic case, with uncorrelated observations, this determinant takes the form

$$
\operatorname{det}(M)=g(\sigma, \gamma, \alpha, \theta) x^{2(\beta 1+\beta 2)}\left(\delta^{\beta_{1}}-\delta^{\beta_{2}}\right)^{2}
$$

\begin{tabular}{|c|c|c|c|c|}
\hline & & $t^{*}$ & & $d^{*}$ \\
\hline$\beta_{1}<0$ & $\begin{array}{l}\beta_{2}<0 \\
\beta_{2}>0\end{array}$ & & $\tau_{1}$ & $\min \left\{\frac{-\operatorname{In}\left(\beta_{2}\right)-\operatorname{In}\left(-\beta_{1}\right)}{\beta_{1}-\beta_{2}}, \tau_{2}-\tau_{1}\right\}$ \\
\hline \multirow{2}{*}{$\beta_{1}>0$} & $\begin{array}{l}\beta_{2}>0 \\
\beta_{2}<0\end{array}$ & & $\begin{array}{l}\tau_{1} \\
\tau\end{array}$ & $\begin{array}{r}\tau_{2}-\tau_{1} \\
\tau_{2}-\tau_{1}\end{array}$ \\
\hline & $\beta_{2}>0$ & $\max \left\{\tau_{2}-\frac{\operatorname{In}\left(\beta_{1}\right)-\operatorname{In}\left(\beta_{2}\right)}{\beta_{1}-\beta_{2}}\right.$ & $\left.\tau_{1}\right\}$ & $\min \left\{\frac{-\operatorname{In}\left(\beta_{1}\right)-\operatorname{In}\left(-\beta_{2}\right)}{\beta_{1}-\beta_{2}}, \tau_{2}-\tau_{1}\right\}$ \\
\hline
\end{tabular}

where $g$ is a function that does not depend on $x$ nor $\delta$. First of all let us assume $\left|\beta_{1}\right|>\left|\beta_{2}\right|$

Table 5. $D$-optimal two-point designs $\left\{t^{*}, t^{*}+d^{*}\right\}$ for model (3) and design space $\left[\tau_{1}, \tau_{2}\right]$ in the uncorrelated case when the condition $\left|\beta_{1}\right|>\left|\beta_{2}\right|$ is assumed 


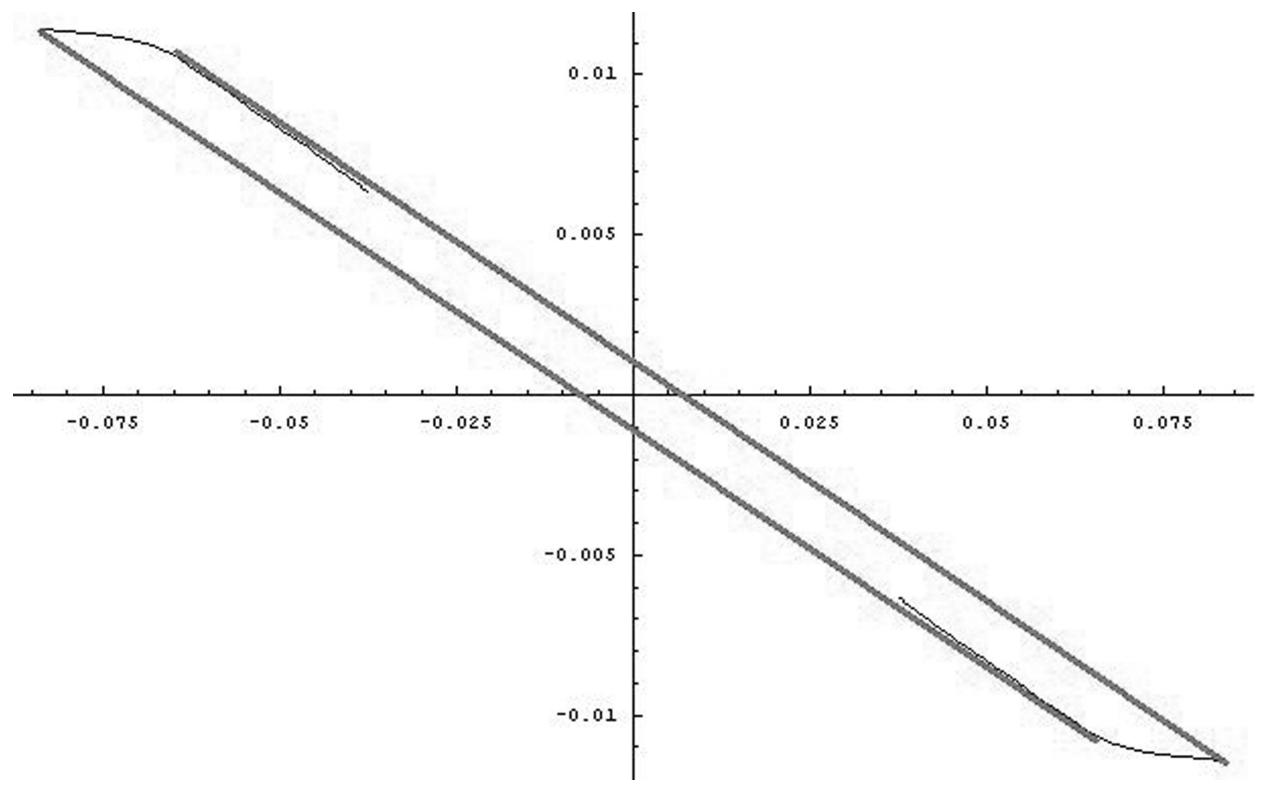

Figure 5. Elfving's set for $I=1000, p=5$

and let us split the interesting part of the determinant in $x^{2(\beta 1+\beta 2)}$ and $\left(\delta^{\beta_{1}}-\delta^{\beta_{2}}\right)^{2}$, that from now on will be $D_{11}$ and $D_{12}$ respectively. We can distinguish two main situations:

- If $\beta_{1}<0$ the exponent of $x$ is negative and $D_{11}$ will be maximized by taking $x$ to be the less possible value, that is, the initial point of the design interval, $e^{\tau_{1}}$. If $\beta_{2}>0, D_{12}$ will

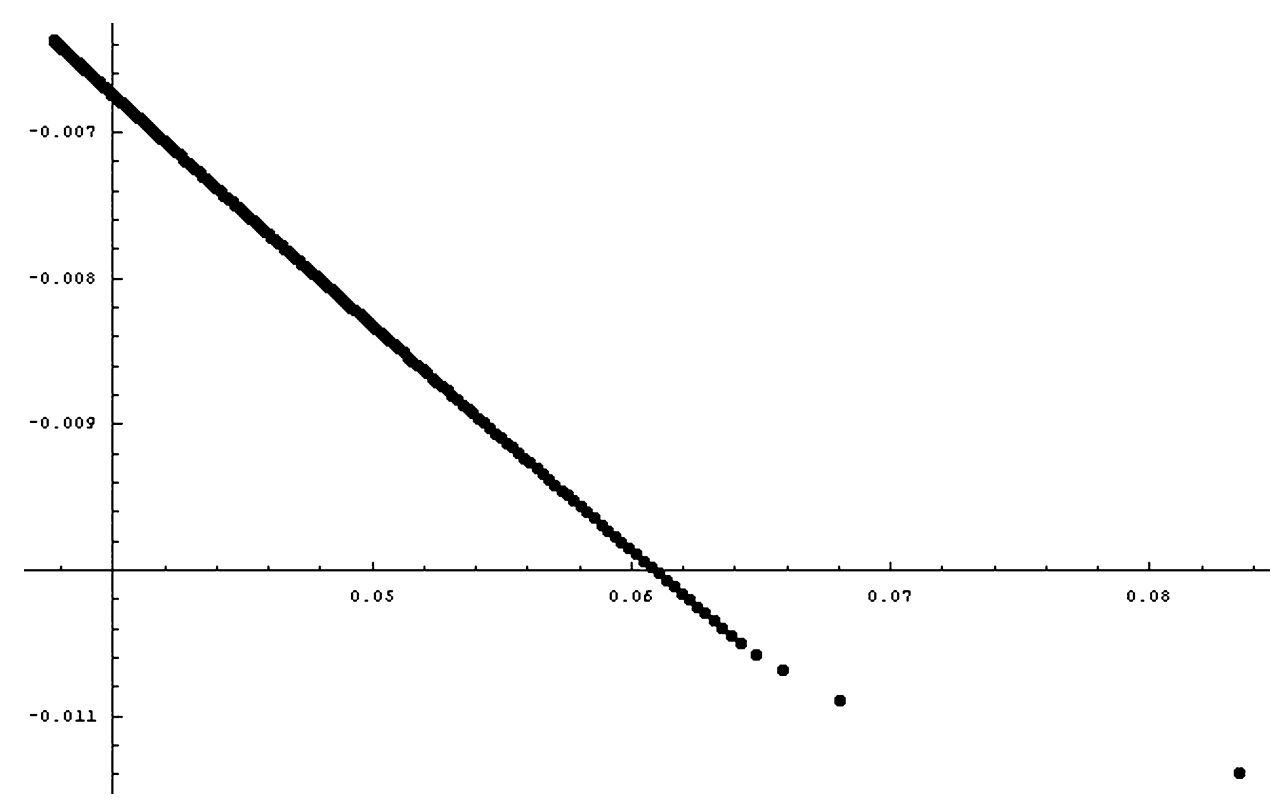

Figure 6. Curvature of the linearized model. Elfving's loci for $\Delta t=0.5$ 
get the maximum value for the greatest $\delta$ we can take, that is $e^{\tau_{2}} / x$. When $\beta_{2}$ is also negative the optimal point for $\delta$ maximizing $D_{12}$ is

$$
\delta^{*}=\left(\frac{\beta_{2}}{\beta_{1}}\right)^{\frac{1}{\beta_{1}-\beta_{2}}}
$$

- If $\beta_{1}>0$ then both $D_{11}$ and $D_{12}$ are maximized for the highest values of the variables $x$ and $\delta$, thus the upper bound for $x \delta, e^{\tau_{2}}$, is reached. Then, using the equality $x \delta=e^{\tau_{2}}$ in equation (4) we obtain for $\delta^{*}$ in the case $\beta_{2}>0$ the inverse expression of the one in equation (5). That means that

$$
x^{*}=e^{\tau_{2}}\left(\frac{\beta_{2}}{\beta_{1}}\right)^{\frac{1}{\beta_{1}-\beta_{2}}}
$$

corresponds to the $x$-value that gives the local maximum. When $\beta_{2}<0$ there is no local maximum, the determinant increases with $\delta$ in the design interval and therefore $\delta^{*}=e^{\tau_{2}-\tau_{1}}$, and consequently $x^{*}=e^{\tau_{1}}$.

Let us consider now the covariance matrix

$$
\Sigma=\sigma^{2} e^{t} p^{2}\left(\begin{array}{cc}
1 & e^{-\rho t} \\
e^{-\rho t} & 1
\end{array}\right)
$$

that takes into account that the variance increases with $t$ and $p$. The latter is due to the fact that the bigger the particles are, the more easily they are eliminated, having smaller values for the retention and making the measurement instruments not so accurate. Now, using the mappings we have seen before, the determinant of the information matrix can be written as

$$
\operatorname{det}(M)=g(\sigma, \alpha, \gamma, \theta) x^{2\left(\beta 1+\beta_{2}-1\right)} \frac{\delta^{2 \rho}\left(\delta^{\beta_{1}}-\delta^{\beta_{2}}\right)^{2}}{\delta^{2 \rho}-1}
$$

$\alpha=\left(\alpha_{1}, \alpha_{2}, \alpha_{3}\right)^{T}, \gamma=\left(\gamma_{1}, \gamma_{2}, \gamma_{3}\right)^{T}$, with $g$ that does not depend on $x$. When $d$ is large, $\delta^{2 \rho}=e^{2 d \rho}>>1$, and the determinant can be approximated by $x^{2\left(\beta 1+\beta_{2}-1\right)}\left(\delta^{\beta_{1}}-\delta^{\beta_{2}}\right)^{2}$. The same study can now be made for this expression, getting quite similar results to the ones for the uncorrelated case. For instance, we will have

$$
d^{*}=\frac{\ln \left(-\beta_{2}\right)-\ln \left(-\beta_{1}\right)}{\beta_{1}-\beta_{2}}
$$

if $\beta_{1}<0$ and $\beta_{2}<0$, or

$$
d^{*}=\frac{\ln \left(\beta_{1}-1\right)-\ln \left(\beta_{2}-1\right)}{\beta_{1}-\beta_{2}}
$$

when both parameters are greater than 1 .

\section{Discussion}

The measurement system only has the capability to detect the isotope when the quantity of this isotope reaches a value usually called the Lower Limit of Detection (LLD). This means that after a number of days, depending on $I$, the bioassays make no sense for a second measurement potentially lower than the LLD. In this paper, no limit was 
assumed on the number of days for the second measurement. From a practical point of view the results obtained here are feasible. Nevertheless, a theoretical second optimal time lower than the LLD would mean that the second bioassay must be taken at the LLD at the most.

To illustrate this, let us assume that a counter measures $140 \mathrm{~Bq}$ in the lungs at time $t=0.5$. Using the original model for $p=5$, an input of $\hat{I}=140 / f_{1}(0.5,5)=2104$ may directly be estimated. For example, for uranium enriched $5 \%$ in ${ }^{235} \mathrm{U}$ there is a LLD of $92 \mathrm{~Bq}$, i.e. under this quantity it is not possible to detect the retention. In this example this happens after $t=50.6$ days. The designs given in this paper do not depend on the input, but in this very real case measurements taken after 50 days do not make sense as they are demanded for example in the designs of Table 4 for $\rho=1$ or greater. The optimal design for those cases forces the second measurement to be taken at LLD $-\varepsilon$, being $\varepsilon$ a number defined by the practitioner. Nevertheless there is here an open line of research to investigate in a better way the situation at the LLD with a suitable distribution.

\section{Acknowledgement}

This work has been supported by the grant from Junta de Castilla y León SA004/01.

\section{Appendix}

The form of the original model is

$$
\begin{aligned}
I( & \frac{0.0128067}{e^{0.170111 p}}-0.0024983 e^{-0.0878945 p-10.0001 t}+0.0124915 e^{-0.0878945 p-2.0001 t} \\
& +0.0388835 e^{-0.170111 p-0.0201 t}+0.0768815 e^{-0.170111 p-0.0011 t} \\
& +0.00699301\left(\frac{0.0212844}{e^{4.35327 p}}+\frac{0.00920991}{e^{0.147244 p}}\right) \\
& +\frac{0.00123877\left(\left(0.0212844 / e^{4.35327 p}\right)+\left(0.00920991 / e^{0.147244 p}\right)\right)}{e^{102.1 t}} \\
& +\frac{0.00100101\left(\left(0.0212844 / e^{4.35327 p}\right)+\left(0.00920991 / e^{0.147244 p}\right)\right)}{e^{100.13 t}} \\
& -\frac{0.251008\left(\left(0.0212844 / e^{4.35327 p}\right)+\left(0.00920991 / e^{0.147244 p}\right)\right)}{e^{10.0001 t}} \\
& +\frac{1.24001\left(\left(0.0212844 / e^{4.35327 p}\right)+\left(0.00920991 / e^{0.147244 p}\right)\right)}{e^{2.0001 t}} \\
& +\frac{1.00201\left(\left(0.0212844 / e^{4.35327 p}\right)+\left(0.00920991 / e^{0.147244 p}\right)\right)}{e^{0.0301 t}} \\
& +0.00699301\left(\frac{-0.0110839}{e^{1.11147 p}}+\frac{0.0110839}{e^{0.123578 p}}\right)
\end{aligned}
$$




$$
\begin{aligned}
& +\frac{0.992009\left(\left(-0.0110839 / e^{1.11147 p}\right)+\left(0.0110839 / e^{0.123578 p}\right)\right)}{e^{10.0001 t}} \\
& +\frac{0.999002\left(\left(-0.0110839 / e^{1.11147 p}\right)+\left(0.0110839 / e^{0.123578 p}\right)\right)}{e^{0.0301 t}} \\
& -\frac{0.0012475\left(\left(0.0212844 / e^{4.35327 p}\right)+\left(0.00920991 / e^{0.147244 p}\right)\right)^{2}}{e^{102.1 t}\left(\left(0.0212844 / e^{4.35327 p}\right)+\left(0.00920991 / e^{0.147244 p}\right)+\left(0.0100737 / e^{0.0878945 p}\right)\right)} \\
& +0.00699301\left(\frac{-0.0171738}{e^{0.566783 p}}+\frac{0.0171738}{e^{0.0577835 p}}\right) \\
& +\frac{0.992009\left(\left(-0.0171738 / e^{0.566783 p}\right)+\left(0.0171738 / e^{0.0577835 p}\right)\right)}{e^{10.0001 t}} \\
& +\frac{0.0171567 e^{0.181361 p}-0.0171567 e^{0.690361 p}-0.0171567 e^{1.16925 p}+0.0171567 e^{1.67825 p}}{e^{10.0001 t}\left(1 . e^{0.748144 p}+1.54943 e^{1.29283 p}-1 . e^{1.73604 p}-1.54943 e^{1.80183 p}\right)} \\
& +\frac{0.0110729 e^{0.871722 p}-0.0221457 e^{1.85961 p}+0.0110729 e^{2.84751 p}}{e^{10.0001 t}\left(1 . e^{1.98319 p}+1.54943 e^{2.52788 p}-1 . e^{2.97109 p}-1.54943 e^{3.03688 p}\right)} \\
& +\frac{-0.0125796 e^{0.147244 p}-0.00544327 e^{4.35327 p}}{e^{2.0001 t}\left(1 . e^{0.235138 p}+0.432707 e^{4.44117 p}+0.473291 e^{4.50051 p}\right)} \\
& +\frac{0.00251591 e^{0.147244 p}+0.00108865 e^{4.35327 p}}{e^{10.0001 t}\left(1 . e^{0.235138 p}+0.432707 e^{4.44117 p}+0.473291 e^{4.50051 p}\right)} \\
& +\frac{0.00531579 e^{0.382382 p}+0.00460036 e^{4.58841 p}}{e^{10.0001 t}\left(1 . e^{4.73565 p}+0.432707 e^{8.94168 p}+0.473291 e^{9.00103 p}\right)} \\
& \left.+\frac{-0.0265789 e^{0.382382 p}-0.0230018 e^{4.58841 p}-0.00497652 e^{8.79444 p}}{e^{2.0001 t}\left(1 . e^{4.73565 p}+0.432707 e^{8.94168 p}+0.473291 e^{9.00103 p}\right)}\right)
\end{aligned}
$$

This is a condensed way to give it. Written as a single fraction will need several pages.

\section{References}

Anderson, D. H. (1983) Compartmental Modeling and Tracer Kinetics. Lecture notes in biomathematics 50 (Springer-Verlag: Berlin).

Elfving, G. (1952) Optimum allocation in linear regression theory, Annals of Statistics, 23, pp. 255-262.

International Commission on Radiological Protection (1994) Human Respiratory Tract Model for Radiological Protection. ICRP Publication 66 (Oxford: Pergamon Press).

López-Fidalgo, J. \& Sánchez, G. (2005) Statistical criteria to establish bioassay programs, Health Physics, 89(4), pp. 333-338.

López-Fidalgo, J. \& Rodríguez-Díaz, J.M. (2004) Elfving method for computing c-optimal designs in more than two dimensions, Metrika, 59, 235-244.

Müller, W.G. \& Stehlík, M. (2004) An example of D-optimal designs in the case of correlated errors, COMPSTAT'2004 Symposium (New York: Physica-Verlag) (to appear).

Sánchez, G. \& López-Fidalgo, J. (2003) Mathematical techniques for solving analytically large compartmental systems, Health Physics, 85(2), pp. 184-193.

Skrable, K.W. (1974) A general equation for the kinetics of linear first-order phenomena and suggested applications, Health Physics, 27, pp. 155-157. 